\title{
Activated leukocyte cell adhesion molecule regulates the interaction between pancreatic cancer cells and stellate cells
}

\author{
WEI-WEI ZHANG ${ }^{1}$, SHU-HUI ZHAN ${ }^{1}$, CHANG-XIN GENG $^{1}$, XIN SUN ${ }^{1}$, \\ MERT ERKAN $^{2}$, JÖRG KLEEFF ${ }^{3}$ and XIANG-JUN XIE ${ }^{1}$ \\ ${ }^{1}$ Department of Gastroenterology, Qingdao Municipal Hospital, Qingdao, Shandong 266071, P.R. China; \\ ${ }^{2}$ Department of Surgery, Koc University School of Medicine, Istanbul 34450, Turkey; \\ ${ }^{3}$ Department of Surgery, Technical University of Munich, D-80333 Munich, Germany
}

Received August 30, 2015; Accepted July 1, 2016

DOI: $10.3892 / \mathrm{mmr} .2016 .5681$

\begin{abstract}
Activated leukocyte cell adhesion molecule (ALCAM/CD166) is a transmembrane glycoprotein that is involved in tumor progression and metastasis. In the present study, the expression and functional role of ALCAM in pancreatic cancer cells and pancreatic stellate cells (PSCs) was investigated. Tissue specimens were obtained from patients with pancreatic ductal adenocarcinoma $(n=56)$ or chronic pancreatitis $(\mathrm{CP} ; \mathrm{n}=10)$, who underwent pancreatic resection, and from normal pancreatic tissue samples $(n=10)$. Immunohistochemistry was used to analyze the localization and expression of ALCAM in pancreatic tissues. Subsequently, reverse transcription-quantitative polymerase chain reaction and immunoblotting were applied to assess the expression of ALCAM in pancreatic cancer Panc-1 and T3M4 cells, as well as in PSCs. An enzyme-linked immunosorbent assay was used to measure ALCAM levels in cell culture medium stimulated by hypoxia, tumor necrosis factor (TNF)- $\alpha$ and transforming growth factor- $\beta$. Silencing of ALCAM was performed using ALCAM small interfering (si)RNA and immunocytochemistry was used to analyze the inhibition efficiency. An invasion assay and a cell interaction assay were performed to assess the invasive ability and co-cultured adhesive potential of Panc-1 and T3M4 cells, as well as PSCs. Histologically, ALCAM expression was generally weak or absent in pancreatic cancer cells, but was markedly upregulated in PSCs in pancreatic cancer tissues. ALCAM was highly expressed in PSCs from CP tissues and PSCs surrounding pancreatic intraepithelial neoplasias, as well as in pancreatic cancer cells. ALCAM mRNA was highly expressed in PSCs, with a low to moderate expression in T3M4 and Panc-1 cells. Similar
\end{abstract}

Correspondence to: Dr Xiang-Jun Xie, Department of Gastroenterology, Qingdao Municipal Hospital, 5 Donghai Middle Road, Qingdao, Shandong 266071, P.R. China

E-mail: xiangjunxie2005@126.com

Key words: activated leukocyte cell adhesion molecule, CD166, pancreatic cancer cells, pancreatic stellate cells, microenvironment to the mRNA expression, immunoblotting demonstrated that ALCAM protein levels were high in PSCs and T3M4 cells, but low in Panc- 1 cells. The expression of TNF- $\alpha$ increased, while hypoxia decreased the secretion of ALCAM in pancreatic cancer Panc-1 and T3M4 cells, and also in PSCs. Silencing of ALCAM by siRNA revealed no significant alteration in the invasion of pancreatic cancer cells, however, it inhibited the invasive ability of PSCs, and decreased the interaction between Panc-1 cells and PSCs. In conclusion, ALCAM is upregulated in PSCs of pancreatic cancer tissues, suggesting a potential role of ALCAM in regulating pancreatic cancer cell-PSC interactions.

\section{Introduction}

Activated leukocyte cell adhesion molecule (ALCAM/CD166) is a $105 \mathrm{kDa}$ transmembrane glycoprotein and a member of the cell surface immunoglobulin superfamily. It was initially identified in thymic epithelial cells and activated leukocytes $(1,2)$. ALCAM mediates cell-cell homophilic (ALCAM-ALCAM) and heterophilic (ALCAM-CD166) interactions. Deregulation of ALCAM is associated with the development and malignant progression of various types of cancer, including colon, gastric, liver, lung, prostate, pancreatic cancer, breast carcinomas and melanoma (3-11). Furthermore, it is a marker for cancer stem cells in colon and prostate cancer $(12,13)$. However, the exact role of ALCAM during the tumorigenesis of different types of malignant tumor remains to be elucidated. It was proposed that ALCAM may promote the survival of breast cancer cells by inhibiting apoptosis and autophagy (14). Evidence has also suggested that ALCAM may suppress the migration and invasion of cancer cells by controlling the activity of matrix metalloproteinases (15). For example, the expression of ALCAM is associated with the suppression of breast cancer cell invasion (16). Furthermore, ALCAM-negative pancreatic cancer cells demonstrated stronger invasive and migratory activities compared with ALCAM-positive cancer cells (17). Our previous study demonstrated that silencing ALCAM caused no affect on cell growth or invasion in Su86.86 pancreatic cancer cells, but significantly reduced cell adhesion and increased pancreatic cancer cell resistance towards chemotherapeutic agents (4). However, the underlying mechanism remains to be elucidated. 
In the present study, the expression of ALCAM in pancreatic cancer cells and pancreatic stellate cells (PSCs) was analyzed, and the role of ALCAM in the growth, proliferation, invasion and cell-cell interaction of pancreatic cancer cells and PSCs was further investigated.

\section{Materials and methods}

Tissue specimens and cell cultures. Tissue specimens were obtained from patients who underwent pancreatic resection. The patients underwent surgery for a range of pancreatic diseases, including pancreatic ductal adenocarcinoma (PDAC; $n=56)$ and chronic pancreatitis $(\mathrm{CP} ; \mathrm{n}=10)$. Normal pancreatic tissue samples $(n=10)$ were obtained during resection for tumor infiltration of the periampullary area by another malignancy (i.e. colon cancer) or metastasis to the pancreas by kidney tumors that required resection of healthy pancreatic tissue. Tissue collection was approved by the Ethics Committees of the Technical University of Munich (Munich, Germany) and the University of Heidelberg (Heidelberg, Germany). Written informed consent was obtained from all patients.

Freshly removed tissues were fixed in $4 \%$ paraformaldehyde for $24 \mathrm{~h}$ and embedded in paraffin for histological analysis. A portion of the tissue samples was preserved in RNAlater (Ambion Europe Ltd., Huntingdon, Cambridgeshire, UK) or snap-frozen in liquid nitrogen immediately upon surgical removal, and maintained at $-80^{\circ} \mathrm{C}$ until use. Pancreatic cancer cell lines (Panc-1 and T3M4) were obtained from American Type Culture Collection (Manassas, VA, USA) and grown in RPMI-1640 medium (Invitrogen; Thermo Fisher Scientific, Inc., Waltham, MA, USA) supplemented with $10 \%$ fetal calf serum (FCS; Invitrogen; Thermo Fisher Scientific, Inc.) and $100 \mathrm{U} / \mathrm{ml}$ penicillin and streptomycin (complete medium) at $37^{\circ} \mathrm{C}$ in a $5 \% \mathrm{CO}_{2}$ humidified atmosphere. PSCs between passages 3 and 6 were cultured in a 1:1 ( $\mathrm{vol} / \mathrm{vol})$ mixture of low-glucose (1,000 mg/l) Dulbecco's modified Eagle's medium with Ham's F12 medium (Invitrogen; Thermo Fisher Scientific, Inc.) supplemented with $10 \%$ FCS, L-glutamine ( $2 \mathrm{mmol} / \mathrm{l})$, penicillin/streptomycin and amphotericin, as previously described (18-21).

Reverse transcription-quantitative polymerase chain reaction $(R T-q P C R)$. All reagents and equipment for mRNA and cDNA preparation were purchased from Roche (Mannheim, Germany). The forward and reverse primer sequences were as follows: ALCAM sense, 5'-TAG CAG GAA TGC AAC TGT GG-3'; ALCAM anti-sense, 5'-CGC AGA CAT AGT TTC CAG-3'. mRNA was prepared by automated isolation using the MagNA Pure LC instrument and isolation kit I (for cells) and kit II (for tissues). RNA was reverse-transcribed into cDNA using the cDNA synthesis kit for RT-PCR (AMV), according to the manufacturer's protocol. qPCR was performed on a LightCycler 480 Real-Time PCR system (Roche Diagnostics, Basel, Switzerland) with the Light Cycler Fast Start DNA SYBR Green kit. The PCR program consisted of an initial denaturation cycle $\left(10 \mathrm{~min}\right.$ at $\left.95^{\circ} \mathrm{C}\right)$ followed by 40 cycles of denaturation $\left(15 \mathrm{sec}\right.$ at $\left.95^{\circ} \mathrm{C}\right)$ and annealing and elongation $\left(60 \mathrm{sec}\right.$ at $\left.60^{\circ} \mathrm{C}\right)$. The relative number of specific transcripts was normalized against the levels of cyclophilin B and hypoxanthine guanine phosphoribosyltransferase, and the data were analyzed using the $2^{-\Delta \Delta \mathrm{Cq}}$ method (22).

Immunohistochemistry. Immunohistochemistry was performed using the Dako Envision System (Dako Cytomation $\mathrm{GmbH}$, Hamburg, Germany). Consecutive paraffin-embedded tissue sections (3-5 $\mu \mathrm{m}$ thick) were deparaffinized and rehydrated using routine methods (23). Antigen retrieval was performed by pretreatment of the slides in citrate buffer (pH 6.0) in a microwave oven for $10 \mathrm{~min}$. Endogenous peroxidase activity was quenched by incubation in deionized water containing $3 \%$ hydrogen peroxide at room temperature for $10 \mathrm{~min}$. Following blocking of non-specific reactivity with diluted normal goat serum, the tissue sections were incubated with rabbit anti-human ALCAM polyclonal antibodies (4 $\mu \mathrm{g} / \mathrm{ml} ; 1: 1,000$, Santa Cruz Biotechnology, Inc., Santa Cruz, CA, USA) at $4^{\circ} \mathrm{C}$ overnight. The tissue sections were subsequently incubated with horseradish peroxidase (HRP)-linked goat anti-rabbit antibodies (1:5,000; Dako GmbH, Hamburg, Germany), followed by reaction with diaminobenzidine and counterstaining with Mayer's hematoxylin. In addition, to confirm the specificity of the primary antibodies, tissue sections were incubated in the absence of the primary antibodies and with negative control rabbit immunoglobulin $\mathrm{G}$ (1:50; Santa Cruz Biotechnology, Inc.).

Immunocytochemistry. The cells (Panc-1, T3M4 and PSCs) were transfected with control or ALCAM small interfering (si)RNA for $48 \mathrm{~h}$, trypsinized and seeded onto slides for $12 \mathrm{~h}$. Following washing three times with phosphate-buffered saline (PBS), the cells were incubated with $4 \%$ paraformaldehyde for $10 \mathrm{~min}, 30 \mathrm{mM}$ glycine/PBS for $5 \mathrm{~min}$ and $0.1 \%$ Triton $\mathrm{X}-100$ for $5 \mathrm{~min}$. After washing three times with PBS, the cells were incubated with $3 \% \mathrm{H}_{2} \mathrm{O}_{2}$ for $10 \mathrm{~min}$, followed by incubation with the primary antibody diluted in universal block DAKO (ALCAM, 1:50; keratin, 1:500, Santa Cruz Biotechnology, Inc.) for $1 \mathrm{~h}$. Following washing with Tris-buffered saline (TBS)/bovine serum albumin (BSA; Carl Roth GmbH \& Co. KG, Karlsruhe, Germany) and Tween $20(0.05 \%)$, the secondary antibody labeled with HRP (1:1,000, Chemicon, Hofheim, Germany) was added, followed by color reaction and counterstaining as in immunohistochemistry.

Enzyme-linked immunosorbent assay (ELISA). The ALCAM ELISA kit was used (R\&D Systems, Wiesbaden-Nordenstadt, Germany) to detect ALCAM levels in the serum and tissue culture medium at room temperature. Briefly, 96-well Nunc Immuno plates (Nunc, Roskilde, Denmark) were coated overnight with $100 \mu \mathrm{l}(2 \mu \mathrm{g} / \mathrm{ml})$ of ALCAM capture antibodies (1:500; R\&D Systems, Inc., Wiesbaden-Nordenstadt, Germany) in PBS (pH 7.0). PBS with $0.05 \%$ Tween 20 was used as the washing solution. Non-specific binding sites were blocked with $300 \mu$ l blocking buffer (1\% BSA in PBS) for $1 \mathrm{~h}$ at $37^{\circ} \mathrm{C}$. Either recombinant human ALCAM or serum/cell culture supernatant (100 $\mu \mathrm{l} /$ well) were added and incubated for $2 \mathrm{~h}$ at $37^{\circ} \mathrm{C}$. Following washing, $100 \mu \mathrm{l}$ of biotin-conjugated goat anti-human ALCAM detection antibodies $(50 \mathrm{ng} / \mathrm{ml})$ were added into each well and incubated for $2 \mathrm{~h}$ at room temperature. HRP-conjugated streptavidin (100 $\mu \mathrm{l})$ 1:200 diluted 
A

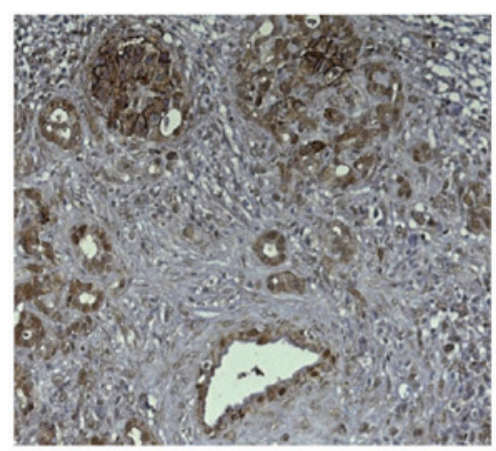

B

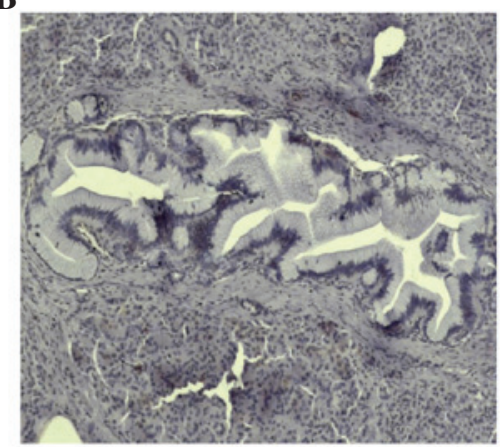

C

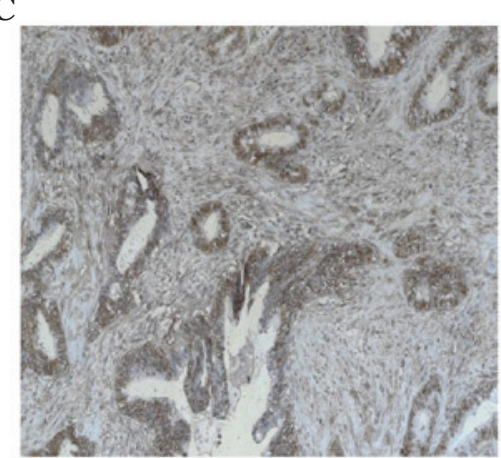

Figure 1. ALCAM expression and localization in pancreatic tissues and cells. (A) Immunohistochemistry of ALCAM demonstrated strong staining in ductal and acinar cells in chronic pancreatitis as well as in surrounding PSCs. (B) No ALCAM staining was found in pancreatic intraepithelial neoplasia. (C) Minimal ALCAM staining was detected in the membrane and cytoplasm of pancreatic cancer cells, (B and C) however, strong staining was detected in PSCs. ALCAM, activated leukocyte cell adhesion molecule; PSCs, pancreatic stellate cells. Magnification, x200.

in PBS was added to each well and incubated for $20 \mathrm{~min}$ at $37^{\circ} \mathrm{C}$. Following washing with PBS with Tween 20 three times, $100 \mu \mathrm{l}$ of 1:1 mixed TMB substrate reagent $\mathrm{A}$ and reagent $\mathrm{B}$ (BD Biosciences, San Diego, CA, USA) were added for $20 \mathrm{~min}$ at $37^{\circ} \mathrm{C}$. Colorimetric reactions were stopped by adding $50 \mu \mathrm{l}$ of $2 \mathrm{~N} \mathrm{H}_{2} \mathrm{SO}_{4}$ and analyzed using a microplate reader at 450 and $570 \mathrm{~nm}$ for correction.

Immunoblotting. Cultured pancreatic cancer cells and PSCs were lysed in ice-cold lysis buffer containing $20 \mathrm{mM}$ Tris- $\mathrm{HCl}$ (pH 7.4), 150 mM NaCl, 1\% Triton X-100, 2.5 mM sodium pyrophosphate and one tablet EDTA-free protease inhibitor cocktail (Roche) for $30 \mathrm{~min}$. Cell lysates were collected following centrifugation at $15,000 \mathrm{x} \mathrm{g}$ for $10 \mathrm{~min}$ at $4^{\circ} \mathrm{C}$. The total protein $(20 \mu \mathrm{g})$ was loaded onto $10 \%$ polyacrylamide gels and transferred onto polyvinylidene difluoride membranes. The membranes were blocked with $20 \mathrm{ml} \mathrm{TBS,} \mathrm{5 \%} \mathrm{skim}$ milk and $0.05 \%$ Tween-20 for $1 \mathrm{~h}$, and then incubated with rabbit anti-human ALCAM polyclonal antibodies (1:200; Santa Cruz Biotechnology, Inc.) or anti-Erk2 (1:2,500; Santa Cruz Biotechnology, Inc.) overnight at $4^{\circ} \mathrm{C}$. The membranes were washed three times with $0.05 \%$ Tween-20-TBS and incubated with HRP-conjugated anti-rabbit antibody (1:2,500; Chemicon) for $1 \mathrm{~h}$ at room temperature. Signals were detected using the enhanced chemiluminescence system (Amersham Life Science, Buckinghamshire, UK).

Hypoxia. Panc-1, T3M4 and PSC cells were exposed to hypoxic conditions of $0.75 \% \mathrm{O}_{2}, 10 \% \mathrm{CO}_{2}$ and $89.25 \% \mathrm{~N}_{2}$, as described previously (24). The medium was switched to serum-free medium (Invitrogen; Thermo Fisher Scientific, Inc., Waltham, MA, USA) prior to subjecting the cells to hypoxia. Control cultures were grown in serum-free medium under normoxia in a $5 \% \mathrm{CO}_{2}$ incubator (Forma Scientific Co., Marietta, $\mathrm{OH}$, USA). After incubation for $48 \mathrm{~h}$, supernatants were collected for ALCAM assay using ELISA.

siRNA transfection. Human ALCAM specific siRNA (sense, GCC CGA UGG CUC CCC AGU A; antisense, UAC UGG GGA GCC AUC GGG C) (14) was purchased from Qiagen (Hilden, Germany). The cells were grown to 50-70\% confluence. siRNA transfection was performed with HiPerFect transfection reagent (Qiagen), according to the manufacturer's instructions. The final concentration of the control and specific siRNA was $5 \mathrm{nM}$. The efficacy of the siRNA transfection was ascertained by immunoblot analysis and ELISA after $72 \mathrm{~h}$ of transfection.

Invasion assay. To assess cell migration in vitro, Transwell migration chambers with an $8 \mu \mathrm{m}$ pore size (BD Biosciences) were used and reconstituted with $600 \mu 1$ serum-free RPMI-1640 medium in the top and bottom chambers for 24 h. The Panc 1, T3M4 and pancreatic stellate cells were trypsinized and seeded into the top chamber at a density of $2.5 \times 10^{4}$ cells/well in $600 \mu \mathrm{l}$ RPMI-1640 containing $10 \%$ FCS. Following incubation at $37^{\circ} \mathrm{C}$ for $20 \mathrm{~h}$, the cells remaining attached to the upper surface of the filters were carefully removed with cotton swabs, while cells that reached the underside of the chamber were stained with hematoxylin and eosin and counted under a microscope in five random fields at a magnification of $\times 200$.

Cell interaction assay. Panc-1 or T3M4 cells in RPMI-1640 supplemented with $10 \%$ FCS and PSCs in a 1:1 ( $\mathrm{vol} / \mathrm{vol})$ mixture of low glucose (1,000 mg/l) Dulbecco's modified Eagle's medium with Ham's F12 medium containing with $10 \%$ FCS were transfected with ALCAM siRNA or control siRNA for 2 days. The tumor cells and PSCs were trypsinized, counted, mixed and seeded directly for co-culture at a density of 4,000 cells/well in 24-well plates. After 2 days of culture, immunocytochemistry using keratin as a specific marker for cancer cells was performed to analyze the interaction between tumor cells (Panc-1 and T3M4) and PSCs. Cell-cell interactions were counted randomly in five areas under the microscope and calculated as a percentage (interaction number/total number of cells). All assays were performed in triplicate and repeated three times.

Statistical analysis. The data are presented as the mean \pm standard error of the mean for in vitro assays, and median and individual data for the RT-qPCR and ELISA results, unless indicated otherwise. Statistical analysis was performed using SPSS 17.0 software (SPSS Inc., Chicago, IL, USA). The Mann-Whitney U test and the Kruskal-Wallis test were 
A

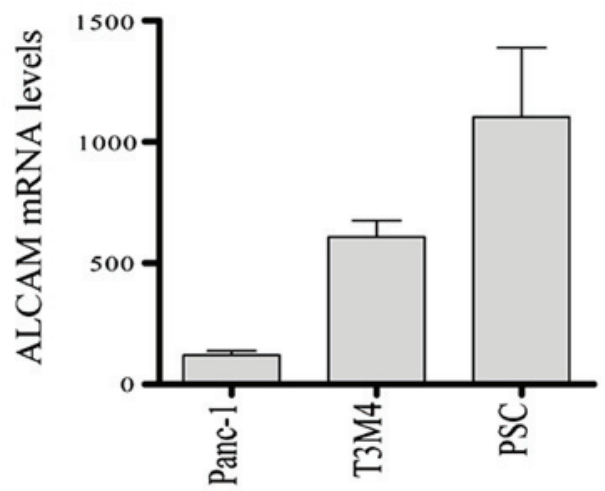

B

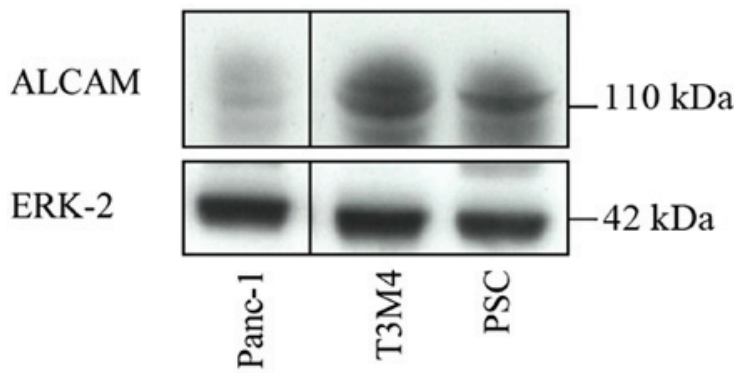

Figure 2. Differential expression of ALCAM in pancreatic cancer cells and PSCs. (A) Reverse transcription-quantitative polymerase chain reaction analysis of ALCAM mRNA levels in the pancreatic cancer cell lines Panc-1 and T3M4, as well as in PSCs. RNA input was normalized against the average expression of hypoxanthine-guanine phosphoribosyltransferase and cylophilin $\mathrm{B}$, and presented as the copy number/ $\mu 1 \mathrm{cDNA}$. Immunoblotting analysis was performed to detect ALCAM protein expression in Panc-1 and T3M4 cells, and also in PSCs. (B) Equal loading of the protein samples was confirmed using an ERK-2 antibody. ALCAM, activated leukocyte cell adhesion molecule; PSCs, pancreatic stellate cells; ERK-2, extracellular signal-regulated kinase-2.

utilized, and groups were compared using Dunn's multiple comparison test. $\mathrm{P}<0.05$ was considered to indicate a statistically significant difference. The mean difference between groups was estimated with a 95\% confidence interval.

\section{Results}

ALCAM expression and localization in pancreatic tissues. Our previous study (4) demonstrated that ALCAM was expressed on the membrane of islet cells in the normal pancreas whereas normal pancreatic ducts were negative for ALCAM. ALCAM was expressed in ductal and acinar cells in CP tissues. Furthermore, ALCAM expression was generally low in PDAC, while membranous or cytoplasmic ALCAM expression was found in certain types of tumor (9). The present study demonstrated strong ALCAM expression in PSCs of CP tissues (Fig. 1A), and PSCs surrounding pancreatic intraepithelial neoplasias (Fig. 1B), as well as in pancreatic cancer cells (Fig. 1C).

ALCAM expression in pancreatic cancer cells and PSCs. A previous study demonstrated that ALCAM was expressed in pancreatic cancer cell lines (9). The present study compared the expression of ALCAM in pancreatic cancer Panc-1 and T3M4 cells with its expression in PSCs. As shown in Fig. 2A,

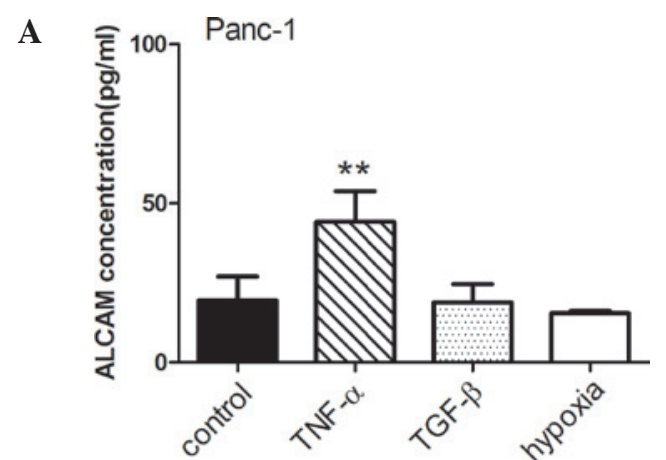

$\mathbf{B}$

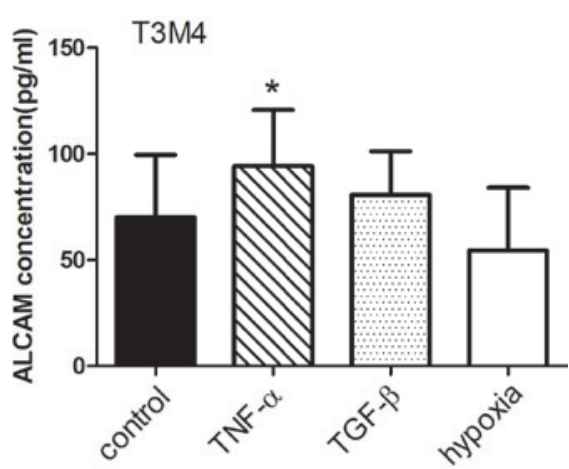

C

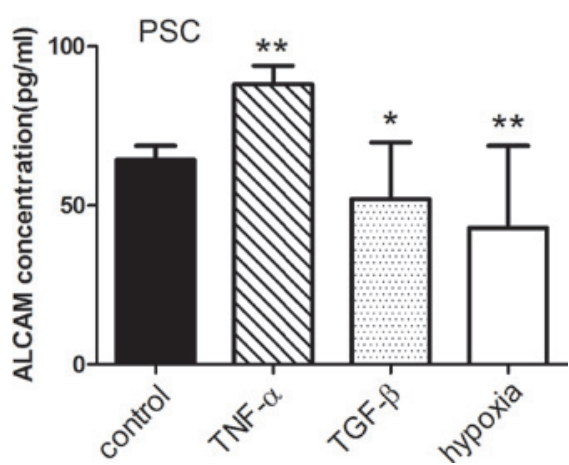

Figure 3. ALCAM levels following stimulation with TNF- $\alpha$, TGF- $\beta$ and hypoxia. Enzyme-linked immunosorbent assay analysis of the levels of ALCAM was performed, as described in the Materials and methods. TNF- $\alpha$, TGF- $\beta$ and hypoxia were used to stimulate (A) Panc-1 and (B) T3M4 cells, and also (C) PSCs. ALCAM levels were significantly increased by TNF- $\alpha$ in (A) Panc-1 ( $\mathrm{P}<0.001)$, (B) T3M4 ( $\mathrm{P}=0.003)$ and (C) PSCs $(\mathrm{P}<0.001)$, whereas ALCAM levels were significantly decreased by hypoxia in PSCs $(\mathrm{P}<0.001)$. ${ }^{*} \mathrm{P}<0.05 ;{ }^{* *} \mathrm{P}<0.001$. TNF- $\alpha$, tumor necrosis factor- $\alpha$; TGF- $\beta$, transforming growth factor- $\beta$; ALCAM, activated leukocyte cell adhesion molecule; PSCs, pancreatic stellate cells.

ALCAM mRNA was highly expressed in PSCs, while it was low to moderately expressed in T3M4 and Panc-1 cells. Similar to mRNA expression, western blot analysis demonstrated that ALCAM protein levels were high in PSCs and T3M4 cells, but low in Panc-1 cells (Fig. 2B).

Soluble levels of ALCAM are regulated by tumor necrosis factor (TNF)- $\alpha$, transforming growth factor (TGF)- $\beta$ and hypoxia. To assess the secretion of ALCAM following stimulation in pancreatic cells, Panc-1 and T3M4 cells, and PSCs were treated with TNF- $\alpha$, TGF- $\beta$ and hypoxia for $48 \mathrm{~h}$, and ALCAM protein levels were detected in cell culture supernatant by ELISA. The results demonstrated that ALCAM levels 
$\mathbf{A}$
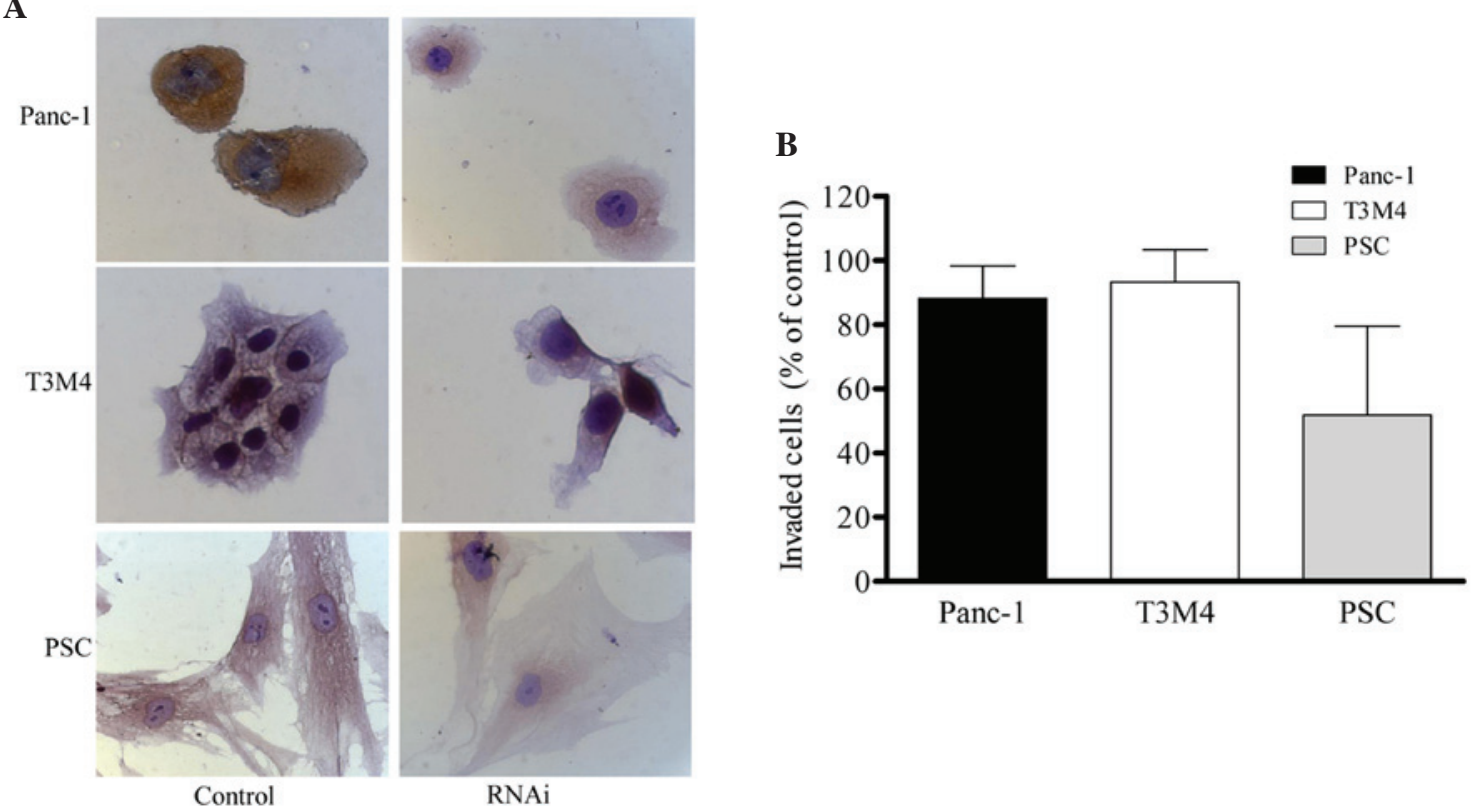

Figure 4. Effects of ALCAM on the invasion of Panc-1 and T3M4 cells, and PSCs. (A) ALCAM expression following transfection with ALCAM siRNA and siRNA control in Panc-1 and T3M4 cells, and PSCs for $48 \mathrm{~h}$ using immunocytochemistry. An in vitro cell invasion assay was performed to evaluate the effects of ALCAM silencing on the invasion of Panc-1 and T3M4 cells, and PSCs. Cells were transfected with ALCAM siRNA or control siRNA for 48 h. (B) The data are shown are the percentages compared with each control group and were obtained from three independent experiments. ALCAM, activated leukocyte cell adhesion molecule; PSCs, pancreatic stellate cells; siRNA, small interfering RNA; RNAi, RNA interference.

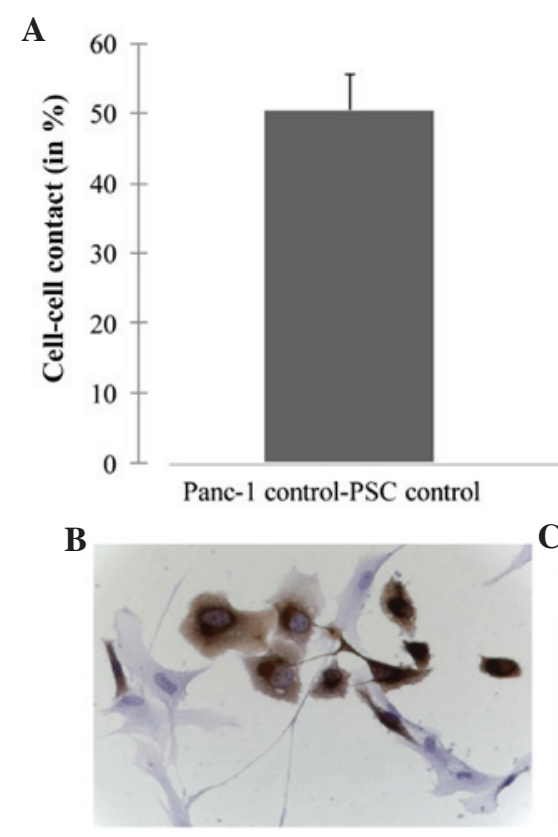

Panc-1 control-PSC control

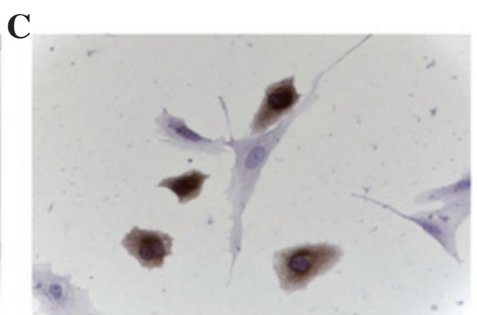

Panc-1 RNAi-PSC RNAi

Figure 5. Detection of tumor-stroma interactions in co-cultured Panc-1 cells and PSCs. Following transfection with ALCAM siRNA for 48 h, Panc-1 cells and PSCs were seeded, as described in the Materials and methods. (A) Cell-cell interaction was counted randomly in five areas under the microscope and calculated as a percentage (interaction number/total number of cells). Representative images for the (B) control group and (C) ALCAM RNAi transfection group are shown. ALCAM, activated leukocyte cell adhesion molecule; PSCs, pancreatic stellate cells; RNAi, RNA interference.

were significantly increased by TNF- $\alpha$ in Panc-1 $(\mathrm{P}<0.001)$, T3M4 ( $\mathrm{P}=0.003)$ and PSCs $(\mathrm{P}<0.001)$, while ALCAM levels were significantly decreased by hypoxia in PSCs $(\mathrm{P}<0.001)$. Following treatment with TGF- $\beta$, ALCAM levels did not change in Panc-1 cells, increased in T3M4 cells $(\mathrm{P}=0.043)$ and decreased in PSCs ( $\mathrm{P}=0.01$; Fig. 3).
ALCAM promotes PSC invasion. As previously demonstrated by our group, ALCAM silencing did not affect pancreatic cancer cell growth or invasion but significantly reduced cell adhesion in Su86.86 pancreatic cancer cells (4). To assess the role of ALCAM in the regulation of invasion of pancreatic cells and PSCs, ALCAM was knocked down using siRNA in Panc-1 and 
T3M4 cells, as well as in PSCs. Immunocytochemistry demonstrated that ALCAM was predominantly expressed in the cytoplasm in Panc-1 and PSC cells, and in the membrane of T3M4 cells. Transfection of these cells with ALCAM siRNA for $48 \mathrm{~h}$ resulted in a decrease in ALCAM expression (Fig. 4A). ALCAM silencing by siRNA did not affect the invasion of Panc-1 and T3M4 cells, but resulted in 50\% inhibition of invasion of PSCs ( $\mathrm{P}=0.047$; Fig. 4B).

ALCAM silencing results in decreased interaction between Panc-1 cells and PSCs. A previous study demonstrated that ALCAM is important in the regulation of tumor cell-stromal cell interactions (25). To assess whether silencing of ALCAM alters the interaction between tumor and stromal cells, Panc-1 and PSCs were co-cultured. Silencing of ALCAM by siRNA led to a decreased interaction between Panc-1 cells and PSCs (Fig. 5).

\section{Discussion}

The molecular functions of ALCAM in the tumorigenesis of different types of cancer are largely unknown. In the present study, immunohistochemistry demonstrated that ALCAM was only partially expressed in the membrane and cytoplasm of pancreatic cancer cells, which was consistent with the results of a previous study by Hong et al (4). ALCAM expression is generally variable in pancreatic cancer cells (17). In the present study, RT-qPCR and immunoblot analyses revealed that the expression of ALCAM was higher in PSCs compared with that in Panc-1 and T3M4 pancreatic cancer cells, suggesting that ALCAM may be more important in PSCs compared with in pancreatic cancer cells.

It was reported that ALCAM protected breast cancer cells against apoptosis and autophagy, suggesting that ALCAM may promote tumorigenesis (14). However, another study demonstrated that ALCAM suppressed breast cancer cell invasion, suggesting inhibition of tumorigenesis at the late stages (16). In addition, $\mathrm{CD}_{166^{+}}$pancreatic cancer cells are strongly tumorigenic, while CD166 pancreatic cancer cells exhibit comparatively stronger invasive and migratory activities (16). These data suggested that ALCAM may either promote or suppress tumorigenesis. However, the effect of ALCAM on PSCs is unknown. Notably, the results of the present study demonstrated that ALCAM silencing by siRNA decreased the invasive ability of PSCs, which are a component of the pancreatic tumor microenvironment. ALCAM shedding would release ALCAM into the tumor environment and circulation to exert its function. When the malignant environment was mimicked in vitro using TNF- $\alpha$, TGF- $\beta$ and hypoxia, ALCAM shedding was significantly induced by TNF- $\alpha$ from pancreatic cancer cells and PSCs, while it was decreased by hypoxia and TGF- $\beta$, particularly in PSCs. The results are consistent with the observation that ALCAM is a cytokine-regulated cell adhesion molecule (26). This indicated that the expression of ALCAM is regulated by an altered tumor microenvironment.

The tumor microenvironment is important in the progression, invasion and metastasis of cancer cells $(27,28)$. ALCAM is variably expressed in stromal and tumor cells, and its expression is affected by the tumor microenvironment. Hong et al reported an association between CD166 and adhesiveness (4).
Adhesiveness may cause the functional differences between $\mathrm{CD}^{266^{+}}$and CD166 cells. Thus, it may be hypothesized that ALCAM-ALCAM interactions facilitate stromal-cancer cell adhesiveness. In support of this hypothesis, in the present study, ALCAM silencing by siRNA did not significantly alter the proliferation of pancreatic cancer cells, but decreased the invasive ability of PSCs. Furthermore, co-culture experiments of PSCs with pancreatic cancer cells demonstrated that silencing of ALCAM in PSCs and Panc-1 cells resulted in decreased tumor cell-PSC adhesiveness. It is well known that once tumor growth has reached a critical mass, the metastatic spread of tumor cells is dependent on their dissociation from the primary tumor and migration towards the systemic circulation. Primary tumors with invasive properties usually exhibit reduced intercellular adhesion, which allows cells to break away from the parental cell mass. Thus, it is possible that ALCAM may indirectly regulate the metastatic potential of pancreatic cancer cells by modulating tumor-stroma interactions.

In conclusion, ALCAM is upregulated in PSCs of pancreatic cancer tissues, promotes PSC invasion and increases the interaction between Panc-1 cells and PSCs, suggesting a potential role of ALCAM in regulating pancreatic cancer cell-PSC interactions.

\section{Acknowledgements}

The present study was supported by the Qingdao Municipal Health Science and Technology Program.

\section{References}

1. Bowen MA, Patel DD, Li X, Modrell B, Malacko AR, Wang WC, Marquardt H, Neubauer M, Pesando JM, Francke U, et al: Cloning, mapping and characterization of activated leukocyte-cell adhesion molecule (ALCAM), a CD6 ligand. J Exp Med 181: 2213-2220, 1995.

2. Patel DD, Wee SF, Whichard LP, Bowen MA, Pesando JM, Aruffo A and Haynes BF: Identification and characterization of a 100-kD ligand for CD6 on human thymic epithelial cells. J Exp Med 181: 1563-1568, 1995.

3. Hansen AG, Arnold SA, Jiang M, Palmer TD, Ketova T, Merkel A, Pickup M, Samaras S, Shyr Y, Moses HL, et al: ALCAM/CD166 is a TGF- $\beta$-responsive marker and functional regulator of prostate cancer metastasis to bone. Cancer Res 74: 1404-1415, 2014.

4. Hong X, Michalski CW, Kong B, Zhang W, Raggi MC, Sauliunaite D, De Oliveira T, Friess H and Kleeff J: ALCAM is associated with chemoresistance and tumor cell adhesion in pancreatic cancer. J Surg Oncol 101: 564-569, 2010.

5. Kozovska Z, Gabrisova V and Kucerova L: Colon cancer: Cancer stem cells markers, drug resistance and treatment. Biomed Pharmacother 68: 911-916, 2014.

6. Penna E, Orso F, Cimino D, Vercellino I, Grassi E, Quaglino E, Turco E and Taverna D: miR-214 coordinates melanoma progression by upregulating ALCAM through TFAP2 and miR-148b downmodulation. Cancer Res 73: 4098-4111, 2013.

7. Piao D, Jiang T, Liu G, Wang B, Xu J and Zhu A: Clinical implications of activated leukocyte cell adhesion molecule expression in breast cancer. Mol Biol Rep 39: 661-668, 2012.

8. Tachezy M, Zander H, Wolters-Eisfeld G, Müller J, Wicklein D, Gebauer F, Izbicki JR and Bockhorn M: Activated leukocyte cell adhesion molecule (CD166): An 'inert' cancer stem cell marker for non-small cell lung cancer? Stem Cells 32: 1429-1436, 2014.

9. Ye M, Du YL, Nie YQ, Zhou ZW, Cao J and Li YF: Overexpression of activated leukocute cell adhesion molecule in gastric cancer is associated with advanced stages and poor prognosis and miR-9 deregulation. Mol Med Rep 11: 2004-2012, 2015. 
10. Yu W, Wang J, Ma L, Tang X, Qiao Y, Pan Q, Yu Y and Sun F: CD166 plays a pro-carcinogenic role in liver cancer cells via inhibition of FOXO proteins through AKT. Oncol Rep 32: 677-683, 2014.

11. Zhao Z, Lu P, Zhang H, Xu H, Gao N, Li M and Liu C: Nestin positively regulates the $\mathrm{Wnt} / \beta$-catenin pathway and the proliferation, survival and invasiveness of breast cancer stem cells. Breast Cancer Res 16: 408, 2014.

12. Dalerba P, Dylla SJ, Park IK, Liu R, Wang X, Cho RW, Hoey T, Gurney A, Huang EH, Simeone DM, et al: Phenotypic characterization of human colorectal cancer stem cells. Proc Natl Acad Sci USA 104: 10158-10163, 2007.

13. Rajasekhar VK, Studer L, Gerald W, Socci ND and Scher HI: Tumour-initiating stem-like cells in human prostate cancer exhibit increased NF- $\mathrm{B}$ B signalling. Nat Commun 2: 162, 2011.

14. Jezierska A, Matysiak W and Motyl T: ALCAM/CD166 protects breast cancer cells against apoptosis and autophagy. Med Sci Monit 12: BR263-BR273, 2006.

15. Lunter PC, van Kilsdonk JW, van Beek H, Cornelissen IM, Bergers M, Willems PH, van Muijen GN and Swart GW: Activated leukocyte cell adhesion molecule (ALCAM/CD166/MEMD), a novel actor in invasive growth, controls matrix metalloproteinase activity. Cancer Res 65: 8801-8808, 2005.

16. Jezierska A, Olszewski WP, Pietruszkiewicz J, Olszewski W, Matysiak W and Motyl T: Activated Leukocyte Cell Adhesion Molecule (ALCAM) is associated with suppression of breast cancer cells invasion. Med Sci Monit 12: BR245-BR256, 2006.

17. Fujiwara K, Ohuchida K, Sada M, Horioka K, Ulrich CD III, Shindo K, Ohtsuka T, Takahata S, Mizumoto K, Oda Y and Tanaka M: CD166/ALCAM expression is characteristic of tumorigenicity and invasive and migratory activities of pancreatic cancer cells. PLoS One 9: e107247, 2014.

18. Erkan M, Adler G, Apte MV, Bachem MG, Buchholz M, Detlefsen S, Esposito I, Friess H, Gress TM, Habisch HJ, et al: StellaTUM: Current consensus and discussion on pancreatic stellate cell research. Gut 61: 172-178, 2012

19. Erkan M, Kleeff J, Gorbachevski A, Reiser C, Mitkus T, Esposito I, Giese T, Büchler MW, Giese NA and Friess H: Periostin creates a tumor-supportive microenvironment in the pancreas by sustaining fibrogenic stellate cell activity. Gastroenterology 132: 1447-1464 2007.
20. Erkan M, Reiser-Erkan C, Michalski CW, Deucker S, Sauliunaite D, Streit S, Esposito I, Friess H and Kleeff J: Cancer-stellate cell interactions perpetuate the hypoxia-fibrosis cycle in pancreatic ductal adenocarcinoma. Neoplasia 11: 497-508, 2009.

21. Zhang W, Erkan M, Abiatari I, Giese NA, Felix K, Kayed H, Büchler MW, Friess H and Kleeff J: Expression of extracellular matrix metalloproteinase inducer (EMMPRIN/CD147) in pancreatic neoplasm and pancreatic stellate cells. Cancer Biol Ther 6: 218-227, 2007.

22. Abiatari I, Esposito I, Oliveira TD, Felix K, Xin H, Penzel R, Giese T, Friess H and Kleeff J: Moesin dependent cytoskeleton remodeling is associated with an anaplastic phenotype of pancreatic cancer. J Cell Mol Med 14: 1166-1179, 2009.

23. Kayed H, Kleeff J, Kolb A, Ketterer K, Keleg S, Felix K, Giese T, Penzel R, Zentgraf H, Büchler MW, et al: FXYD3 is overexpressed in pancreatic ductal adenocarcinoma and influences pancreatic cancer cell growth. Int J Cancer 118: 43-54, 2006.

24. Erkan M, Kleeff J, Esposito I, Giese T, Ketterer K, Büchler MW, Giese NA and Friess H: Loss of BNIP3 expression is a late event in pancreatic cancer contributing to chemoresistance and worsened prognosis. Oncogene 24: 4421-4432, 2005

25. Behnan J, Isakson P, Joel M, Cilio C, Langmoen IA, Vik-Mo EO and Badn W: Recruited brain tumor-derived mesenchymal stem cells contribute to brain tumor progression. Stem Cells 32: 1110-1123, 2014.

26. Levesque MC, Heinly CS, Whichard LP and Patel DD: Cytokine-regulated expression of activated leukocyte cell adhesion molecule (CD166) on monocyte-lineage cells and in rheumatoid arthritis synovium. Arthritis Rheum 41: 2221-2229, 1998.

27. Habisch H, Zhou S, Siech M and Bachem MG: Interaction of stellate cells with pancreatic carcinoma cells. Cancers (Basel) 2: 1661-1682, 2010.

28. Wilson JS, Pirola RC and Apte MV: Stars and stripes in pancreatic cancer: Role of stellate cells and stroma in cancer progression. Front Physiol 5: 52, 2014. 